\section{PERFECT NUMBERS}

John Cosgrave

(Carysfort)

A natural number of is said to be perfect 19 the sum of all the divisors of a equals $2 \mathrm{~m}$. Thus, 6 and 28 are perfect, because $1,2,3$ and 6 are the factors of $6 ; 1,2,4,7,14$ and 28 are those of 28 and $1+2+3+6=12=2 \times 6$.

$$
1+2+4+7+14+28=56=2 \times 28 .
$$

One could say that $m$ is perfect if the sum of all the factors of in, less than $m$, equals m. Thus

$$
1+2+3=6
$$

and $1+2+4+7+14=28$.

Anyone tho did the calculations required to find which integers $m$ ith $f \leqslant 1000$ (say), were perfect, would find only three examples, namely 6,28 and 496 . These numbers have the following "prime factorization"

$$
\begin{aligned}
6 & =2^{1} \times 3^{1} \\
28 & =2^{2} \times 7^{1} \\
496 & =2^{4} \times 31^{1}
\end{aligned}
$$

$3,7,31$ are the only proper odd factors of 6,28 and 496 respectively, and they are alsoprimes - not only that, for 1 t 1 s also striking that they are each one less than a power of 2 , and also these powers of two are in each case one more than the corresponding power of 2 occurring in the prime decompositions of 6,28 and 496 (namely $2^{1}, 2^{2}, 2^{3}$ ) so one s led to guess that
Theorem 1 If the number $\left(2^{n}-1\right)$ is prime, then the number $2^{\mathrm{n}-1}\left(2^{\mathrm{n}}-1\right)$ is perfect.

Proof Let $p=\left(2^{n}-1\right)$ and suppose $p$ is prime. Then the factors of $2^{n-1} \cdot p$ are as follows:

$$
1,2,2^{2}, \ldots, 2^{n-1} \quad \text { (the1r sum } 1 \text { s }\left(2^{n}-1\right) \text { ) }
$$

and $p, 2 p, 2^{2} p, \ldots, 2^{n-1} p$ (their sum is $\left(2^{n}-1\right) p$ ).

Thus the sum of all the factors of $2^{n-1} \cdot\left(2^{n}-1\right)$ is:

$$
\left(2^{n}-1\right)+\left(2^{n}-1\right) p, \quad \text { i.e. } \quad\left(2^{n}-1\right)(1+p) \text {. This equals }\left(2^{n}-1\right) 2^{n},
$$
which 1 s $2 .\left(2^{n-1} \cdot\right.$ p). So $2^{n-1}\left(2^{n}-1\right)$ is perfect.

Now 1 t could be that $2^{4} p$ is perfect for some prime $p$ other than 31. Lets see what $p$ would have to be 11 ke. Suppose $24 p$ was perfect. then since the factors of $2^{4}$ p are: $1,2,2^{2}, 2^{3}, 2^{4}$ (whose sum is 31 ), and $p, 2 p, 2^{2} p, 2^{3} p, 2^{4} p$ (whose sum is $31 p$ ), It would follow that

$$
\begin{aligned}
31+31 p & =2 \times\left(2^{4} p\right)=32 p \\
\text { 1.e. } 31+31 p & =32 p \\
\text { i.e. } \quad 31 & =32 p-31 p=p
\end{aligned}
$$

so $p$ would have to equal 31 after all:

You are now ready for

Theorem 2 If the integer $2^{n-1} \cdot p$ is perfect, where $p$ is an odd prime, then $p=\left(2^{n}-1\right)$.

Proof The factors of $2^{n-1} \cdot p$ are: 


$$
\begin{aligned}
& 1,2,2^{2}, \ldots, 2^{n-1} \quad \text { (whose sum 1s }\left(2^{n}-1\right) \text { ) } \\
& \text { and } p, 2 p, 2^{2} p, \ldots, 2^{n-1} \text { (whose sum 1s }\left(2^{n}-1\right) p \text { ), }
\end{aligned}
$$

and it would follow that:

$$
\begin{aligned}
\left(2^{n}-1\right)+\left(2^{n}-1\right) p & =2 \times\left(2^{n-1} p\right)=2^{n} p \\
\text { 1.e. } \quad\left(2^{n}-1\right)+\left(2^{n}-1\right) p & =2^{n} p . \\
\text { 1.e. } \quad\left(2^{n}-1\right) & =2^{n} p-\left(2^{n}-1\right) p=p \\
\text { So we have } \quad\left(2^{n}-1\right) & =p .
\end{aligned}
$$

Theorem 1 and 2 were known to Euclid and tell us that the only even perfect numbers of the form $2^{n-1} p$ are those for which $p=\left(2^{n}-1\right)$. These perfect numbers are said to be of Euclid type. The theorem of Euler (18th century) states that the only even perfect numbers are those of Euclid type (so there are none of the form, say, $2^{4} p^{2} q^{3}$, where $p, q$ are odd primes).

What we now wish to ask is this, when is the number $\left(2^{n}-1\right)$ a prime number. These numbers are called the Mersenne numbers $\left(M_{n}, M_{n}=\left(2^{n}-1\right)\right)$ after the 17 th century French mathematician Mersenne, (see for example, Volume One of Dicksons Theory of Numbers).

$M_{2}, M_{3}, M_{5}$ and $M_{7}$ (1.e. $3,7,31$ and 127 ) are prime, $M_{4}, M_{6}, M_{8}, M_{9}$ and $M_{10}$ are composite. It 1 s easy to show that $1 f \mathrm{n}$ 1s composite then $M_{n}$ is composite. It was once (wrongly) thought that if $n$ is prime then $M_{n}$ is prime and this is seen to be false for the next case after $M_{7}$ for $M_{11}=\left(2^{n}-1\right)=2047=23 \times 89$.

Theorem 3 if $n$ is composite so also is $M_{n}$. b with $a, b \geqslant 2$. Now $M_{n}=\left(2^{n}-1\right)=\left(2^{a b}-1\right)$

$=\left(2^{a}-1\right)\left(2^{a(b-1)}+\ldots+2^{b}+1\right)$.

Now $a \geq 2 \Rightarrow\left(2^{a}-1\right) \geq\left(2^{2}-1\right)=3$,

and $b \geqslant 2 \Rightarrow\left(2^{a(b-1)}+\ldots+2^{b}+1\right) \geqslant\left(-+2^{2}+1\right)=5$.

Thus $M_{n}$ 1s the product of two integers $\left(2^{4}-1\right)$ and $\left(2^{a(b-1)}+\ldots+2^{b}+1\right)$ netther of which is 1 , and so $M_{n}$ is composite.

So we are now interested in the numbers $M_{2}, M_{3}, M_{5}, M_{7}, M_{11}, M_{13}, M_{17}$, $M_{19}, M_{23}, M_{29}, M_{31}, \ldots$ i.e. the Mersenne numbers with prime suffix. $M_{2}, M_{3}, M_{5}$ and $M_{7}$ were known to be prime by A.D.100, Regius (1536) noted that $M_{11}$ is composite. Cataldi (1603) showed that $M_{13}, M_{17}$ and $M_{19}$ are primes (by checking for primedivisors a la Eratosthenes), but erred in claiming that so also are $M_{23}$ and $M_{29}$ and $M_{37}$ (why did he not say anything about $M_{31}$ ?). These last three were put right by Fermat (1640) who showed that $\left(2^{23}-1\right)$ is divisible by 47 , and that $\left(2^{37}-1\right)$ is divisible by 223 ; and Euler (1732) who showed that $\left(2^{29}-1\right)$ is divisible by 1103 .

In 1644, Mersenne claimed that the f1rst eleven perfect numbers are Given by $2^{p-1}\left(2^{p}-1\right)$ for $p=2,3,5,7,13,17,19,31,67,127,257$; but he erred at least in including 67 and excluding 61,89 and 107 . Laczo (1867) proved that $\left(2^{67}-1\right)$ is composite (without actually exhibiting a, factorization). Cole (1903) gave an explicit factorization of $M_{67}$ which was

$$
\left(2^{67}-1\right) \div 193,707,721 \times 761,838,257,287 .
$$

Euler (1750) showed that $\left(2^{31}-1\right)$ is prime; Lucas (1876) showed that $\left(2^{127}-1\right)$ is prime: 
Burvouahthi (1883) unowed that $\left(2^{81}-1\right)$ 1. prtimo;

Powerw (1911) showed that $\left(2^{80}-1\right)$ 18 prime;

Powers (1914) showed that $\left(2^{107}-1\right)$ is prime.

So all the Meisenne primes (in order) up to $M_{127}$ are:

$M_{2,3,5,7,13,17,19,31,61,89,107,127}$

$M_{127}=170,141,183,460,469,231,731,687,303,715,884,105,727$.

D. Lehiner (1951-52) showed that $\left(2^{521}-1\right),\left(2^{607}-1\right),\left(2^{1279}-1\right)$, $\left(2^{2203}-1\right)$ and $\left(2^{2281}-1\right)$ are primes. Riesel (1958) showed that $\left(2^{3217}-1\right)$ is prime. Harwitz?(1960) showed that $\left(2^{4253}-1\right)$ and $\left(2^{4423}-1\right)$ are primes. Gollies (1964) showed that $\left(2^{9689}-1\right),\left(2^{9941}-1\right)$ and $\left(2^{112,3}-1\right)$ are primes. Tuckermann (1971) showed that $\left(2^{19937}-1\right)$ is prime. Lastly, the 25 th Mersenne prime was discovered on the 30 October 1978 by two 18 year old studente (at the California State Un1versity), Laura N1cke1 and Kurt No11:

$$
M_{21,701}=\left(2^{21,701}-1\right) \text { 1s prime, }
$$

and so,

$$
2^{21,700}\left(2^{21,701}-1\right) \text { to the } 25 \text { th even perfect number. }
$$

Theorem (Euler): Let $p$ be an odd prime, then any prime factor of $M_{p}\left(=\left(2^{p}-1\right)\right)$ must be of the form $(2 n p+1)$ for some integer $n$. Th1s, together wh the observation that if a number $N$ is composite It has a prime factor $\leqslant \sqrt{ }$, , enabled Euler to verify the primaltty of Mp for small values of $p$. To take an example: to check if $\left(2^{13}-1\right)$ is prime, $\left(2^{13}-1\right)=8191$. According to Euler, any prime factor of $\left(2^{13}-1\right)$ muat be of the form $(2 n .13)+1$, 1.e. of the form
$(26 n+1)$. According to Eratosthenes we need only search for prime divisors up to 8191 , which 18 between 90 and 91 . Now when

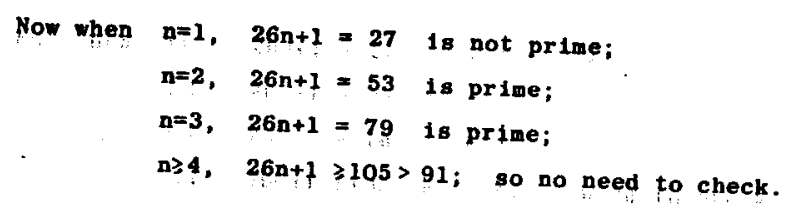

Now $1 t$ only remains to check if 53 or 79 divide 8191 . If neither does, ve know 8191 1s prime. You should check to see.

The Euter test 1nvolves so much calculation for large $p$ as to make his test of no practical value. The following test (the only one used - the only other one, in fact) is the one which has been used since 1876 .

Lucas-Lehper Theorem: Let $p=3,5,7,11,13,17,19, \ldots$ (odd prime) Define the sequence $\left\{a_{n}\right\}$ as follows:

$$
a_{1}=14, a_{2}=a_{1}^{2}-2, \quad a_{3}=a_{2}^{2}-2 \text { etc. }
$$

Then (1) 11 p divides ap-2, $u_{p}$ ts prime.

(1i) $17 \mu_{p}$ does not divide $n_{p-2}, K_{p}$ is composite

Examples When $p=3 . M_{p}=2^{3}-1=7 . \quad a_{p-2}=a_{3-2}=a_{1}=24$ and. here $u_{p}$ divides ap-2. and $\mu_{p}$ (1.e. ?) 1s prime.

When $p=5 . \quad x_{p}=\left(2^{5}-1\right)=31 . \quad p_{p-2}=a_{5-2}=3$. Now $a_{1}=14$. $n_{2}=14^{2}-2=196-2=194 . \quad q_{3}=194^{2}-2=37636-2=37634$, therefore $M_{3}$ divides $a_{3}$, and 31 divides 37634 , therefore $M_{5}$ is prime 
Shorter Solut 1on (uging congruences)

$a_{1}=14, \quad a_{2}=14^{2}-1=196-2=194$.

Now $a_{2}=194 \equiv 8(\bmod 31)$ (since $\left.194=(6.31)+8\right)$

Therefore $a_{2} \equiv 8^{2}(\bmod 31)$

$a_{2}^{2}-2 \equiv 8^{2}-2 \equiv 64-2 \equiv 62 \equiv 0(\bmod 31)$

$a_{3} \equiv O(\bmod 31)$. Therefore $M_{5}$ divides $a_{3}$, and $M_{5}$ is prime.

\section{SECONDARY SCHOOL MATHEMATICS}

\section{T.J. Larfoy}

There hide been duite an amount of discisision for some time now on the content of mathematics courses in secondary schoola. Mainy people teel that there has been a general decline in the level of computational skill and ability to solve problems th students who leave schooi.

Varfous attempts are being made to tdentify the reasons por this and to find remedies. Among the reasons suggested are the following:-

(1) The "New Math",

(2) Over-emphasis on teaching "concepts" and a peeling that as long as the "concepts" are O.k., the answer doesn't matter,

(3) The more abstract presentation of material, particularly geometry, with the result that the students are not taught to relate mathematics to commonsense and experience,

(4) An unwillingness on the part of teachers and pupils to spend large amounts of time going through, perhaps somewhat dull and repetitive routines, in order that students get to know these techniques thoroughly.

At present, there is a great deal of debate going on throughout the world on the value of the so-called New Math. (1.e. sets, relations 